\section{Weiterentwickelte Misch- und Dosieranlage}

Qampf Dosiertechnik präsentierte auf der RMesse Chinaplas 2013, die vom 20. bis zum 23. Mai in Guangzhou, China, stattfand, die Niederdruck-Misch- und Dosieranlage DCCNC 800, die mit zahlreichen technischen Neuerungen aufwartet. Unter anderem verfügt die Anlage über eine verbesserte Materialaufbereitung mit größeren Tanks, sodass Kleingebinde komplett in einem Vorgang umgefüllt werden können.

Der Einsatz dieser Misch- und Dosieranlage empfiehlt sich insbesondere für den zwei- oder dreidimensionalen Materialauftrag von Verguss-, Dichtungs- und Klebstoffsystemen. Im Grundaufbau verfügt die Anlage über eine integrierte Materialaufbereitung und kann wahlweise mit Kolben- oder Zahnradpumpen ausgestattet werden.

Weitere Features sind die modulare Steuerung und die integrierte Prozessüberwachung zur permanenten Kontrolle von Drücken, Füllständen und Drehzahlen. Die Misch- und Dosieranlage kann optional mit einem HD-Spülmittelrückführsystem oder einer HD-Wasserspülung sowie anwendungsabhängigen Automatisierungseinrichtungen ausgestattet werden.

Im Vergleich zum Vorgängermodell verfügt diese Anlage u.a. über eine verbesserte Materialaufbereitung für eine materialschonendere Homogenisierung und eine schnellere Entgasung. Auch die Zugänglichkeit für Wartungsarbeiten an den Dosierpumpen und der Steuerung wurde deutlich verbessert.

Auf der Chinaplas 2013 wurde vor Ort das Applizieren eines Dichtungsschaummaterials demonstriert, das sich durch eine geringe Wasseraufnahme, gute mechanische Festigkeit und hohe Wärmebeständigkeit auszeichnet. Aufgrund der kurzen Klebfreizeit des Materials von weniger als drei Minuten kann das abgedichtete Bauteil schnell weiterverarbeitet werden.

Weitere Infos: www.rampf-dosiertechnik.de

\section{SM Selbstklebetechnik}

Wir entwickeln und fertigen kundenspezifische Stanz- und Druckerzeugnisse rund um das Thema Kennzeichnung und Klebetechnik.

Ab sofort suchen wir Sie als Mitarbeiter/in für die Stellen

\section{Verkaufs-Innendienst/ anwendungstechnische Beratung}

Ihre Aufgabe:

- Kfm. Auftragsabwicklung vom Angebot bis zur Rechnungsstellung

- technische Arbeitsvorbereitung

- Kundenbetreuung am Telefon, im Hause, vor Ort

\section{Anforderungen:}

- sehr gutes technisches Verständnis und kfm.-/PC-Kenntnisse

- hohes Maß an Qualitäts- und Verantwortungsbewußtsein.

\section{Verkaufs-Außendienst/ anwendungstechnische Beratung} Ihre Aufgabe:

- Betreuung des bestehenden Kundenstamms und Kundengewinnung

- Erarbeitung von klebe- und drucktechnischen Problemlösungen

Anforderungen:

- Identifikation mit dem Produkt

- Sie arbeiten selbständig, organisiert und zuverlässig.

\section{Produktionsleiter}

Ihre Aufgabe:

- Verantwortlich für die fach-/termingerechte Abwicklung der Produktionsaufträge

- einschließlich Lager/Versand, Reparatur und Wartung der Produktionsmaschinen,

- 8 Mitarbeiter in Produktion und Versand.

- Aktive Mitarbeit an allen Produktionsarbeitsplätzen

Anforderungen:

- Abgeschlossene technische und/oder kfm./techn. Berufsausbildung

- Erfahrungen/Kenntnisse in der Stanz-/Schneid-/Wickelund Kaschiertechnik

- Ideal wären Erfahrungen im Maschinenbau oder in der Drucktechnik.

- Flexibles Denken und Handeln, Durchsetzungs- und klares Urteilsvermögen

Sie erfüllen unsere Anforderungen am besten wenn Sie über Branchenerfahrung in der Klebetechnik oder einer vergleichbaren Technologie verfügen.

Es erwarten Sie vielseitige, abwechslungsreiche und gut dotierte Aufgabengebiete in einem hochmotivierten Team innerhalb einer überschaubaren Unternehmensgröße.

Wir freuen uns auf Ihre Bewerbung. Senden Sie diese bitte an unsere Email-Adresse: petri@klebeteam.de, zu Händen Herrn Dieter Petri, oder per Post. Nennen Sie uns bitte Ihren Gehaltswunsch und möglichen Eintrittstermin.

www.sm-selbstklebetechnik.de

SM Selbstklebetechnik GmbH \& Co. KG Moosfeldstraße 2

D-82275 Emmering bei München

Tel: 08141-35356-20 